\title{
Conclusión general
}

https://doi.org/10.21830/9789585241466.09

El entrenamiento físico de un militar está condicionado por factores tanto medioambientales como genéticos, pero el determinante más relevante que tiene un impacto significativo en las condiciones de salud y que se ve influenciado por los hábitos, está representado por los estilos de vida. En efecto, mantener una preparación física adecuada se convierte en un impors tante factor de bienestar y salud. El militar que pertenezca a cualquier fuerza de seguridad del Estado necesita per se un buen nivel de preparación, dada las altas demandas de actividad física y mental durante el desarrollo de las labores propias de su profesión, bien sea durante los tiempos de entrenamiento, bien sea en el desarrollo de sus deberes en los diferentes teatros de operaciones.

La vigilancia y monitorización del nivel del acondicionamiento físico en cualquier militar debe ser un hito fundamental, que tiene que ser evaluado y analizado en el desempeño operacional, así como los mecanismos para asegurar las destrezas del personal combatiente. Un estado físico adecuado puede proteger al soldado contra noxas estresantes propias del desarrollo de su actividad, lo mismo que una reducción de otras complicaciones ante cualquier tipo de lesión. Es sabido que el entrenamiento intensivo disminuye los factores de riesgo para enfermedades cardiovasculares, llevando a una pérdida del peso corporal por reducción del porcentaje de grasa visceral y a un aumento del nivel cardio-respiratorio. Estos hallazgos se traducen igualmente en una reducción del riesgo de morbi-mortalidad, tras obtener mejoras en el bienestar relacionado con la calidad de vida. 
Debido a que la preparación física ha sido conceptualizada como un constructo multidimensional (resistencia cardio-respiratoria, potencia muscular, resistencia muscular, flexibilidad, composición corporal), su evaluación incluye múltiples baterías y diversas pruebas que han sido elaboradas en laboratorios y centros de alto rendimiento, pero adaptadas a los campos específicos del entrenamiento físico-militar. En este libro se abart caron e implementaron algunas herramientas de medición propias de los centros de investigación en actividad física, como son el uso de la bioimpedancia eléctrica en la determinación de la composición corporal, el uso de plataformas de fuerza y aceleradores inerciales para la cuantificación de la fuerza propulsiva y potencia muscular de miembros superiores e inferiores, lo mismo que la determinación de las asimetrías en las diferentes fases del salto evaluadas. También se usaron pruebas específicas como el test de «Sit and Reach", 90-90 y el test de ELY en la evaluación de la flexibilidad de grupos de deportistas militares. De otra parte, se utilizaron pruebas de campo adaptadas al medio militar y deportivo que son un reflejo indirecto de lo que sucede en un equipo de laboratorio (el uso del test de Cooper con respecto a algunas variables ventilatorias obtenidas mediante un ergoespirómetro).

Dentro de las contribuciones que esta obra aporta para llenar el vacío en el conocimiento acerca del del entrenamiento físico militar, se puede mencionar que es la primera iniciativa de aproximación en la evaluación del nivel de preparación física del militar colombiano. Todos los estudios de esta compilación parten de una rigurosidad científica desde la perspectiva metodológica, que permiten asimismo la reproducibilidad de las pruebas (validez externa). Esto último es la base para que otros investigadores hagan la determinación de los baremos de las variables físicas, fisiológicas y biomecánicas del estado y capacidades físicas de la población militar en el país. Los autores son conscientes que dentro de la ejecución de los trabajos que componen la obra se hallan limitantes en algunos estudios en cuanto al número de la muestra, pero corresponden a grupos poblacionales pequeños. Además, téngase en cuanta que esto abre la posibilidad para que nuevos estudios con muestras más representativas puedan ser contrastados con los resultados aquí obtenidos, mejorando así la validez interna de los mismos. 
Este libro es el primer paso de una serie de investigaciones en rendimiento físico militar y factores asociados a la salud en miembros activos del Ejército Nacional, que buscará primero caracterizar, evaluar y determinar los planes de entrenamiento que optimicen los pilares de la doctrina a nivel institucional. La meta última es alcanzar mejores condiciones físicas para el combatiente, en pro de una mejor calidad de vida y seguridad en el despeńo operacional. 